\title{
Functional Electrical Stimulation with Augmented Feedback Training Improves Gait and Functional Performance in Individuals with Chronic Stroke: A Randomized Controlled Trial
}

\author{
Kyung-Hoon Yu', Kwon-Young Kang ${ }^{2}$ \\ 'Department of Physical Therapy, Division of Health Science, Baekseok University, Cheonan; ${ }^{2}$ Department of Physical Therapy, Division of Health \\ Welfare, Wonkwang Health Science University, Iksan, Korea
}

Purpose: The purpose of this study was to compare the effects of the FES-gait with augmented feedback training to the FES alone on the gait and functional performance in individuals with chronic stroke.

Methods: This study used a pretest and posttest randomized control design. The subjects who signed the agreement were randomly divided into 12 experimental groups and 12 control groups. The experimental groups performed two types of augmented feedback training (knowledge of performance and knowledge of results) together with FES, and the control group performed FES on the TA and GM without augmented feedback and then walked for 30 minutes for 40 meters. Both the experimental groups and the control groups received training five times a week for four weeks.

Results: The groups that received the FES with augmented feedback training significantly showed a greater improvement in single limb support (SLS) and gait velocity than the groups that received FES alone. In addition, timed up and go (TUG) test and six minute walk test (6MWT) showed a significant improvement in the groups that received FES with augmented feedback compared to the groups that received FES alone.

Conclusion: Compared with the existing FES gait training, augmented feedback showed improvements in gait parameters, walking ability, and dynamic balance. The augmented feedback will be an important method that can provide motivation for motor learning to stroke patients.

Keywords: Feedback, Electrical stimulation, Gait, Stroke

\section{INTRODUCTION}

Stroke patients complain of difficulties in their daily lives and participation in their community due to impaired walking ability that commonly follows a stroke. Thus, various intervention methods have been introduced for stroke patients who experience difficulty walking. ${ }^{2}$ Ankle dorsiflexion in the swing phase during walking plays an important role in preventing falls. In addition, weakness or paralysis of the ankle dorsiflexors compensates for excessive flexion of the knee and hip joints or circumduction gait. ${ }^{3}$ To address these problems, studies on functional electrical stimulation (FES) as an intervention to improve walking have been conducted. FES is known to be a positive intervention for recovering motor skills and

Received Feb 28, 2017 Revised Apr 27, 2017

Accepted Apr 30, 2017

Corresponding author Kyung-Hoon Yu

E-mail yukh@bu.ac.kr function for stroke patients who have movement problems due to paralysis because FES can provide repeated sensory feedback. ${ }^{4,5}$ Recent meta-analyses have shown that FES-assisted gait training was more effective at improving walking speed and walking distance than gait training alone. ${ }^{6,7}$ Recent studies of patients with chronic stroke have shown that gait training with FES stimulation of the tibialis anterior (TA) in the swing and the gluteus medius (GM) in the stance significantly improved gait performance and dynamic balance. $^{8,9}$

Adding additional feedback to implicit feedback is called augmented feedback, which is commonly used in clinical practice and can play an important role in motor learning. ${ }^{10,11}$ Augmented feedback can be categorized into the knowledge of results and the

Copylight (C2017 The Korea Society of Physical Therapy

This is an Open Access article distribute under the terms of the Creative Commons Attribution Non-commercial License (Http:// creativecommons.org/license/by-nc/4.o.) which permits unrestricted non-commercial use, distribution, and reproduction in any medium, provided the original work is properly cited. 
knowledge of performance. Knowledge of results refers to achieving a goal using information about the outcome of a movement and the subject is verbally performed the outcome. Knowledge of performance refers to the provision of information about skills that affect the outcome while performing a movement, such as giving feedback to improve the dorsiflexion of the ankle in the swing phase. ${ }^{12}$ Feedbac $\mathrm{k}$ processes that provide information about movement errors and how patients correct these errors can have a positive effect on the improvement of walking ability.

Compared with the positive results of the FES training, there are insufficient studies to guide the FES application to be the best practice. FES-gait training using real-time feedback of muscles may be helpful in the function of the paralyzed legs in stroke patients. ${ }^{13}$ In a case study in which FES was applied to patients with chronic stroke, real-time feedback training of the leg movement on the monitor was improved for the walking speed and stride length in the paretic limb, and was maintained for one month. ${ }^{14}$ Therefore, augmented feedback training in FES-based gait training is necessary for motor learning of gait ability, and physical therapists will be an important clinical meaning for FES application during gait training. ${ }^{15}$

Although previous systematic reviews and meta-analyses have demonstrated that FES is more effective intervention for walking performance than gait training without FES, there is a lack of research for the method of FES training to maximize the therapeutic effect in clinical practice. Therefore, it is necessary to demonstrate the therapeutic effect of application of FES with augmented feedback and the best practice guidelines in the clinical application of FES. The purpose of this study was to compare the effects of the FES with augmented feedback-assisted gait training to gait trainingapplied FES alone on the gait and balance of individuals with chronic stroke.

\section{METHODS}

\section{Subjects}

The stroke patients who signed the agreement were randomly divided into 12 experimental groups and 12 control groups. The inclusion criteria for the subjects were: (1) those whose stroke diagnosis was between six months and three years, (2) who could walk more than $10 \mathrm{~m}$ without the help of assistive devices, (3) who had a manual muscle test score below grade 2 (full range of motion after removing gravity) in their TA and GM, and (4) who scored more than 24 points on the MMSE-K (mini-mental status examinationKorean version) test, (i.e., a person who understands simple instructions or explanations). Patients with severe heart disease, difficulty walking due to orthopedic problems, cerebellar or vestibular dysfunction, or susceptibility to electrical stimulation were excluded. The subjects' general characteristics are shown in Table 1. There were no significant differences between the general characteristics of the FES with augmented feedback groups and the FES alone groups. All participants agreed to join this study and provided written informed consent prior to pre-tests.

\section{Procedure and intervention}

FES electrodes were attached to the subjects' TA and GM in the paretic limb before gait training. The TA electrodes were attached to the lateral muscle belly of the shaft of the tibia about one-third and two-thirds between the knee and ankle. In addition, the GM electrodes were attached $5 \mathrm{~cm}$ below the iliac crest and $3 \mathrm{~cm}$ above the greater trochanter on the line connecting the greater trochanter to the height of the iliac crest. Subjects performed the following process to determine the intensity and response of the FES stimulus. The strength of the FES was set so that ankle dorsiflexion could be

Table 1. General characteristics of participants

\begin{tabular}{lcc}
\hline & FES with augmented feedback group & FES alone group \\
\hline Gender (male/female) & $7 / 5$ & $7 / 5$ \\
Age (year) & $56.42 \pm 8.01$ & $58.92 \pm 6.69$ \\
Post-stroke duration (month) & $12.92 \pm 4.78$ & $14.33 \pm 4.91$ \\
Etiology (infarction/hemorrhage) & $8 / 4$ & $6 / 6$ \\
Side of hemiparesis (right/left) & $6 / 6$ & $5 / 7$ \\
Brunnstrum's stage (stage 3/stage 4) & $7 / 5$ & $7 / 5$ \\
MMSE-K & $28.50 \pm 1.24$ & $28.08 \pm 2.15$ \\
\hline
\end{tabular}

Values are presented as mean \pm standard deviation (SD).

MMSE-K: mini-mental state examination-Korean, FES: functional electrical stimulation. 
at least 10 degrees against the gravity (manual muscle test grade 2 or more) by electric stimulation within the range of no pain in the sitting position. Electrodes of GM set the intensity of the FES stimulation so that hip abduction could be performed at least 20 degrees in a standing position. ${ }^{7}$ In the stance phase, electrical stimulation was delivered to the GM, and in the swing phase, it was delivered alternately to the TA.

The experimental groups performed the FES with augmented feedback-assisted gait training which consisted of knowledge of performance $(\mathrm{KP})$ and knowledge of results $(\mathrm{KR}) .{ }^{10}$ The therapist set goals for the improvement of performance of the TA in the swing phase and the performance of the GM in the stance phase when the subjects walked 30 meters. The subjects performed KP feedback training on movement errors, such as the angle of ankle dorsiflexion in the swing phase and the hip abduction angle or Trendelenburg's sign in the stance phase. In the swing phase, therapists give the subject a verbal feedback: "Raise your ankle with electrical stimulation" or in the stance phase, "Strengthen the muscles of the hip joint with electrical stimulation and raise your body into an upright position”. KR feedback training was defined to information related to the outcome of performance and to achieve gait-related goals (e.g., gait speed or swing/stance time). For example, participants gave feedback on the result of walking. "Let's walk a little faster" or "Move your feet a little further". During the 40 minutes of gait training with FES, physical therapist was provided with information related to KP and KR for stroke patients. The level of difficulty and guidelines were set up by referring to stages of motor learning of Fitts and Posner. ${ }^{16}$ In the first step, the subjects were given many verbal instructions to solve the error through feedback during walking training. In the second stage, feedback was performed before and after gait training during variable practice (eg, different gait speed and stride length). Finally, while performing variable practice, the subjects minimized feedback by correcting errors themselves.

The control groups performed FES on the TA and GM without augmented feedback and then walked for 30 minutes for 40 meters. Both the experimental groups and the control groups trained five times a week for four weeks.

\section{Instrument}

\section{1) Functional electrical stimulation}

This study required FES equipment (Microstim2, Sejin MT, Ko- rea), a foot switch, and four electrodes. The electrical stimulation of the GM was induced in the stance phase, where the pressure of the foot switch attached to the heel is increased, and the stimulation of the TA was induced in the swing phase, where the pressure on the foot switch disappears. The FES was set to biphasic rectangular pulses and pulse duration of $200 \mu s^{9,17}$

\section{Outcome measures}

\section{1) The spatiotemporal parameters}

Spatiotemporal parameters were measured using the OptoGait system (OptoGait, Microgate, Bolzano, Italy), which consisted of five transmitting and five receiving bars. Each bar $(100 \times 8 \mathrm{~cm})$ contained 96 light-emitting diodes. The light transmitted from one bar was blocked by the subject's foot and was recognized as a footprint. Data was extracted at $1,000 \mathrm{~Hz}$ and transmitted to using OptoGait software version 1.10.7.0. This system has shown good concurrent validity between the OptoGait and the GaitRite in older adults and high test-retest reliability (Intraclass corelation coefficient, ICC > 0.79 ) in patients with stroke. ${ }^{18,19}$ In this study, the spatiotemporal parameters were used to measure stride length $(\mathrm{cm})$, step time (sec), and single-limb support (SLS, \% gait cycle) duration in the more paretic side, in addition, double-limb support (DLS, \% gait cycle) duration and gait velocity $(\mathrm{m} / \mathrm{s})$ were used.

\section{2) Clinical measures}

\section{(1) The timed up and go (TUG) test}

The TUG test is a simple test that is commonly used to assess functional balance skills in older adults and people with stroke. ${ }^{20,21}$ The TUG test has excellent interrater and intrarater reliability in individuals with stroke $(\mathrm{ICCs}=0.95) .{ }^{20}$

\section{(2) The six-min walk test (6MWT)}

The 6MWT was designed to assess the walking endurance and functional walking distance in individuals with stroke and patients with cardiorespiratory disease. ${ }^{22}$ Studies using the 6MWT have reported a significant correlation between velocity and lower limb strength, ${ }^{3}$ and the 6MWT was strongly associated with comfortable gait speed. $^{23}$

(3) The lower extremity of the Fugl-Meyer assessment (FMA-LE)

Motor recovery and function of lower limb after hemiplegic 
stroke was measured by the lower extremity of the Fugl-Meyer assessment (FMA-LE). On the FMA-LE, motor function is scored on a three-point scale $(0=$ cannot perform, 1 = partially perform, 2 = fully perform), and the motor scores of the FMA-LE range from 0 to 36 points. ${ }^{24}$ The FMA-LE was a high interrater reliability in individuals with stroke. ${ }^{25}$

\section{Statistical analysis}

The data from this study was analyzed using the SPSS statistical package 18.0 (SPSS, Chicago, IL, USA). The normality of distribution of the data was tested for using the Kolmogorov-Smirnov (KS) test. As a result of KS test $(\mathrm{p}<0.05)$, the nonparametric analysis used to determine statistical significance between and within groups. The comparison between the pre- and posttest in each group used the Wilcoxon matched pairs signed-rank test. To compare the effects of gait training on spatiotemporal parameters and clinical measures between experimental and control groups, the MannWhitney U-test was used. An alpha level of $\mathrm{p}<0.05$ was used to define statistical significance.

\section{RESULTS}

Table 2 shows the spatiotemporal parameters within and between groups. After training, the groups that received FES with augmented feedback training showed a significant improvement in stride length $(z=-2.982, p=0.003)$, SLS $(z=-2.510, p=0.012)$, DLS $(z=$ $-2.511, p=0.012)$, and velocity $(z=-3.062, p=0.002)$. Stride length $(\mathrm{z}=-2.937, \mathrm{p}=0.003)$, and velocity $(\mathrm{z}=-2.640, \mathrm{p}=0.008)$ showed $\mathrm{a}$ significant improvement in the groups that received FES alone. The SLS and velocity showed significantly greater improvement in the groups that received FES with augmented feedback training than the groups that received FES alone.

The groups that received FES with augmented feedback after training showed a significant improvement in their TUG $(\mathrm{z}=-2.394$, $\mathrm{p}=0.017), 6 \mathrm{MWT}(\mathrm{z}=-3.063, \mathrm{p}=0.002)$, and FMA-LE $(\mathrm{z}=-3.274$, $\mathrm{p}=0.001)$. The groups that received FES alone showed significant improvement in the 6MWT $(\mathrm{z}=-3.084, \mathrm{p}=0.002)$, and FMA-LE $(\mathrm{z}=-2.530, \mathrm{p}=0.011)$. The TUG and 6MWT results showed significantly greater improvement in the groups that received FES with augmented feedback training than the groups that received FES alone (Table 3).

\section{DISCUSSION}

This study demonstrated changes on gait, motor function, and functional balance in patients with chronic stroke who received FES-assisted gait training with augmented feedback. The results of this study were: (1) the subjects who applied to the FES with augmented feedback training improved their walking abilities, such as velocity and SLS, compared with those who received FES alone and

Table 2. Comparison of spatiotemporal gait parameters within and between groups $(\mathrm{N}=24)$

\begin{tabular}{|c|c|c|c|c|c|c|}
\hline \multirow{2}{*}{ Variables } & \multicolumn{2}{|c|}{ FES with augmented feedback group } & \multicolumn{2}{|c|}{ FES alone group } & \multirow{2}{*}{ z } & \multirow{2}{*}{$p$} \\
\hline & Pre-intervention & Post-intervention & Pre-intervention & Post-intervention & & \\
\hline Stride length $(\mathrm{cm})$ & $92.18 \pm 23.69$ & $101.64 \pm 22.05^{\star}$ & $86.03 \pm 20.01$ & $91.55 \pm 22.94^{*}$ & -1.848 & 0.068 \\
\hline Single limb support (SLS, \%) & $28.29 \pm 7.30$ & $33.53 \pm 4.17^{\star}$ & $28.93 \pm 8.64$ & $29.59 \pm 7.73$ & -2.021 & $0.045^{+}$ \\
\hline Double limb support (DLS, \%) & $38.39 \pm 13.23$ & $32.35 \pm 4.63^{*}$ & $40.25 \pm 14.38$ & $35.18 \pm 13.15$ & -0.549 & 0.590 \\
\hline Velocity $(\mathrm{cm} / \mathrm{s})$ & $0.74 \pm 0.28$ & $0.85 \pm 0.29^{\star}$ & $0.68 \pm 0.27$ & $0.72 \pm 0.28 *$ & -2.432 & $0.014^{+}$ \\
\hline
\end{tabular}

Values are presented as mean \pm standard deviation (SD).

*Significant differences between pre- and post-test $(p<0.05)$; 'Significant differences between FES with augmented feedback group and FES alone group ( $p<0.05)$.

Table 3. Comparison of clinical measures within and between groups $(\mathrm{N}=24)$

\begin{tabular}{|c|c|c|c|c|c|c|}
\hline \multirow{2}{*}{ Variables } & \multicolumn{2}{|c|}{ FES with augmented feedback group } & \multicolumn{2}{|c|}{ FES alone group } & \multirow{2}{*}{ Z } & \multirow{2}{*}{$\mathrm{p}$} \\
\hline & Pre-intervention & Post-intervention & Pre-intervention & Post-intervention & & \\
\hline TUG & $17.66 \pm 7.58$ & $15.70 \pm 6.28^{\star}$ & $20.63 \pm 13.49$ & $20.39 \pm 13.43$ & -2.258 & $0.024^{+}$ \\
\hline 6MWT & $300.83 \pm 101.33$ & $336.33 \pm 87.53^{*}$ & $279.92 \pm 134.88$ & $294.67 \pm 136.49^{*}$ & -2.831 & $0.004^{+}$ \\
\hline FMA-LE & $25.50 \pm 4.03$ & $26.75 \pm 3.55^{\star}$ & $24.00 \pm 6.71$ & $24.67 \pm 6.65^{\star}$ & -2.181 & 0.068 \\
\hline
\end{tabular}

Values are presented as mean \pm standard deviation (SD).

TUG: timed up and go, 6MWT: six minute walk test, FMA-LE: Fugl-Meyer assessment-lower extremity.

*Significant differences between pre- and post-test $(p<0.05)$; ${ }^{+}$Significant differences between FES with augmented feedback group and FES alone group ( $\left.p<0.05\right)$. 
(2) the FES with augmented feedback training improved the clinical measures (TUG test, 6MWT, and FMA-LE) of motor recovery and balance.

This study has shown FES-augmented feedback training as a more effective and appropriate practice in clinical condition, and would like to provide guidance to physical therapists. Therefore, feedback was applied to external focus of attention, which is easier and more motivational than feedback using self-modifying internal focus of attention. ${ }^{26}$ Although it is a protocol of single subject design, there have been two studies to modify motion using visual feedback and biofeedback studies to improve muscle contraction ability using EMG with FES. In these studies, improvement of walking speed and gait parameters were observed. This study is based on the results that verbal feedback using KR and KP methods has an effect on the autonomy of walking ability and the improvement of motor learning of stroke patients.

Decreases in TA strength in stroke patients causes the impaired control of ankle dorsiflexion in the swing phase and results in a decrease in walking speed and an increase in DLS duration. As the TA weakens, swing time are greatly increased for the foot clearance of the more-affected limb compared with other variables, and the SLS duration of the less-affected limb is increased. ${ }^{27,28}$ Recent studies using FES have reported the enhancement of walking speed, ${ }^{6,9,17,29,30}$ and gait training group that received FES application to the GM and TA significantly improved in gait velocity, and stride length compared to group that received no FES. ${ }^{9}$ As above results, the present study also showed a significant improvement in gait velocity and stride length in both groups. In addition, FES with augmented feedback training improved the SLS and DLS duration compared to values of pre-test. When comparing the differences from pre-test to post-test between two groups, the FES with augmented feedback training was greater improvement in SLS duration and gait velocity than the groups that received the FES alone. Therefore, the group that performed the augmented feedback training might have contributed to improve the activation of GM in the stance phase and the performance skill of ankle dorsiflexion in swing compared to the FES-gait training group.

The FES applied to the TA or peroneal nerve for 12 weeks showed an increase in FMA-LE scores and a decrease in the spasticity of the plantarflexors compared with the general rehabilitation. ${ }^{31}$ The application of FES to the dorsiflexors and plantarflexors for three months in patients with chronic stroke showed improvements in strength and gait performance. ${ }^{32}$ In addition, when chronic stroke patients with foot drops were applied to FES for 30 weeks, the subjects were found to be clinically effective in functional evaluation according to their FMA-LE, TUG test, and 6MWT.33 Above-mentioned studies have shown beneficial to effects from long-term FES application. In the present study, short-term FES application with augmented feedback training also showed a significant effect on clinical measures in the same way as previous ones. Although the long-term studies showed a significant clinical effect, this study also proved that short-term FES application with augmented feedback training can also have a better effect on clinical measures than FES application without augmented feedback training.

This study has several limitations that should be considered to generalize and apply the study's results. Although the short duration (four weeks) of this study were effective at improving walking and clinical measures, this study did not examine whether the effects of the training remained after training. Further studies will require transfer and retention tests to examine the long-term effectiveness of the training and to investigate motor learning on walking performance.

The augmented feedback used in this study was composed of two aspects: feedback on the motion of the ankle and hip joints in the swing and stance phases and feedback on the results of performing the motor skill. The FES application with augmented feedback-assisted gait training showed more significant improvements in gait parameters, functional mobility, and walking endurance compared with FES alone. Therefore, augmented feedback will be a practical guideline as FES-assisted gait training that can provide motivation for motor learning to stroke patients.

\section{REFERENCES}

1. Schmid A, Duncan PW, Studenski S et al. Improvements in speed-based gait classifications are meaningful. Stroke. 2007;38(7):2096-100.

2. Kim SH, Choi JD. The effect of gait training of progressive increasing in body weight support and gait speed on stroke patients. J Korean Soc Phys Ther. 2013:25(5):252-9.

3. Nadeau S, Gravel D, Arsenault AB et al. Plantarflexor weakness as a limiting factor of gait speed in stroke subjects and the compensating role of hip flexors. Clin Biomech. 1999;14:125-35.

4. Howlett OA, Lannin NA, Ada L et al. Functional electrical stimulation improves activity after stroke: A systematic review with meta-analysis. 
Arch Phys Med Rehabil. 2015;96(5):934-43.

5. An SH, Lee YM, Yang KH. Effectiveness of gait training using an electromechanical gait trainer combined with simultaneous functional electrical stimulation in chronic stroke patients. J Koren Soc Phys Ther. 2008; 20(1):41-7.

6. Robbins SM, Houghton PE, Woodbury MG et al. The therapeutic effect of functional and transcutaneous electric stimulation on improving gait speed in stroke patients: A meta-analysis. Arch Phys Med Rehabil. 2006; 87:853-9.

7. Pereira S, Mehta S, McIntyre A et al. Functional electrical stimulation for improving gait in persons with chronic stroke. Top stroke rehabil. 2012; 19(6):491-8.

8. Kim JH, Chung Y, Kim Y et al. Functional electrical stimulation applied to gluteus medius and tibialis anterior corresponding gait cycle for stroke. Gait posture. 2012;36(1):65-7.

9. Chung Y, Kim JH, Cha Y et al. Therapeutic effect of functional electrical stimulation-triggered gait training corresponding gait cycle for stroke. Gait posture. 2014;40(3):471-5.

10. Van Vliet PM, Gabriele W. Extrinsic feedback for motor learning after stroke: what is the evidence? 2006;28:831-40.

11. Seo DK, Oh DW, Lee SH. Effectiveness of ankle visuoperceptual-feedback training on balance and gait functions in hemiparetic patients. J Kor Soc Phys Ther. 2010;22(4):35-41.

12. Winstein CJ, Pohl PS, Cardinale C et al. Learning a partial-weight-bearing skill: effectiveness of two forms of feedback. Phy Ther. 1996;76(9): 985-93.

13. Chen YL, Li YC, Kuo TS et al. The development of a closed-loop controlled functional electrical stimulation (FES) in gait training. J Med Eng Technol. 2001;25(2):41-8.

14. Krishnamoorthy V, Hsu WL, Kesar TM et al. Gait training after stroke: A pilot study combining a gravity-balanced orthosis, functional electrical stimulation, and visual feedback. J Neurol PhysThe. 2008;32(4):192-202.

15. Auchstaetter N, Luc J, Lukye S et al. Physical therapists' use of functional electrical stimulation for clients with stroke: Frequency, barriers, and facilitators. Phys Ther. 2016;96:995-1005.

16. Fitts PM and Posner MI. Human performance. Belmont, CA. Brooks/ Cole. 1967

17. O’Dell MW, Dunning K, Kluding P et al. Response and prediction of improvement in gait speed from functional electrical stimulation in persons with poststroke drop foot. PMR 2014;6:587-601.

18. Lienhard K, Schneider D, Maffiuletti NA. Validity of the Optogait photoelectric system for the assessment of spatiotemporal gait parameters. Med Eng Phys. 2013;35(4):500-4.

19. Lee M, Song C, Lee K et al. Agreement between the spatio-temporal gait parameters from treadmill-based photoelectric cell and the instrument- ed treadmill system in healthy young adults and stroke patients. Med Sci Monit. 2014;20:1210-9.

20. Ng SS, Hui-Chan CW. The timed up \& go test: Its reliability and association with lower-limb impairments and locomotor capacities in people with chronic stroke. Arch Phys Med Rehabil. 2005;86(8):1641-7.

21. Podsiadlo D, Richardson S. The timed "Up \& Go": a test of basic functional mobility for frail elderly persons. J Am Geriatr Soc. 1991;39:1428 .

22. Butland RJ, Pang J, Gross ER et al. Two-, six-, and twelve-minute walk tests in respiratory disease. BMJ (Clinical research ed). 1982;284:1607-8.

23. Kelly JO, Kilbreath SL, Davis GM, et al. Cardiorespiratory fitness and walking ability in subacute stroke patients. Arch Phys Med Rehabil. 2003;84:1780-5.

24. Gladstone DJ, Danells CJ, Black SE. The Fugl-Meyer assessment of motor recovery after stroke: A critical review of its measurement properties. Neurorehabil Neural Repair. 2002;16(3):232-40.

25. Sanford J, Moreland J, Swanson LR et al. Reliability of the Fugl-Meyer assessment for testing motor performance in patients following stroke. Phy Ther. 1993; 73:447-54.

26. McNevin NH, Shea CH, Wulf G. Increasing the distance of an external focus of attention enhances learning. Psycholog Res. 2003;67:22-9.

27 Lin PY, Yang YR, Cheng SJ et al. The relation between ankle impairments and gait velocity and symmetry in people with stroke. Arch Phys Med Rehabil. 2006;87:562-8.

28. Kim JH. Relationship between gait symmetry and functional balance, walking performance in subjects with stroke. J Korean Soc Phys Ther. 2014;26(1):1-8.

29. Kottink AI, Hermens HJ, Nene AV et al. A randomized controlled trial of an implantable 2-channel peroneal nerve stimulator on walking speed and activity in poststroke hemiplegia. Arch Phys Med Rehabil. 2007; 88(8):971-8.

30. Bethoux F, Rogers HL, Nolan KJ et al. The effects of peroneal nerve functional electrical stimulation versus ankle-foot orthosis in patients with chronic stroke: A randomized controlled trial. Neurorehabil Neural Repair. 2014;28(7):688-97.

31. Sabut SK, Sikdar C, Kumar R et al. Functional electrical stimulation of dorsiflexor muscle: Effects on dorsiflexor strength, plantarflexor spasticity, and motor recovery in stroke patients. NeuroRehabilitation. 2011; 29(4):393-400.

32. Embrey DG, Holtz SL, Alon G, et al. Functional electrical stimulation to dorsiflexors and plantar flexors during gait to improve walking in adults with chronic hemiplegia. Arch Phys Med Rehabil. 2010;91:687-96.

33. Kluding PM, Dunning K, O’Dell MW, et al. Foot drop stimulation versus ankle foot orthosis after stroke 30-week outcomes. Stroke. 2013; 44(6):1660-9. 